\title{
VECTOR-BASED ACTIVE SURFACES FOR SEGMENTATION OF DYNAMIC PET IMAGES
}

\author{
Vincent Jaouen $^{1}$, Paulo González $z^{1,3}$, Simon Stute ${ }^{2}$, Denis Guilloteau ${ }^{1}$, Irène Buvat ${ }^{2}$ and Clovis Tauber ${ }^{1}$ \\ ${ }^{1}$ UMRS Inserm U930, Université François Rabelais, Tours, France \\ ${ }^{2}$ IMNC, IN2P3, UMR 8165 CNRS-Paris 7 and Université Paris 11, Orsay, France \\ ${ }^{3}$ Universidad Católica del Maule, Talca, Chile
}

\begin{abstract}
Dynamic PET imaging enables the study of radiotracers concentration kinetics along time. However, PET images suffer from limited spatial resolution, low signal-to-noise ratio, and time dependent contrast between tissues, making segmentation of biological volumes difficult. Deformable models are of great interest due to their inherent boundary continuity, but their applications to PET images still remains challenging due to high sensitivity to noise. To address these limitations, we propose a method to perform 3-D+time dynamic PET image segmentation using parametric active surfaces based on a gradient of the vectorial image. This method takes advantage of both spatial and temporal consistency of the signal along the acquisition. We validate our method using GATE Monte Carlo simulations and compare it with single frame based active surface models. We show significant improvement of several figures of merit.
\end{abstract}

Index Terms - 3-D segmentation, Active surface, Dynamic PET, Vector field convolution

\section{INTRODUCTION}

Positron emission tomography (PET), as a functional imaging modality, allows studies of physiological processes within living organisms. However, PET images suffer from several shortcomings, including limited spatial resolution, time dependent contrast between tissues and intrinsic noise that lowers the quality of the reconstructed images. For these reasons, accurate segmentation of true biological volumes in PET images remains difficult (e.g. brain imaging of neuroinflammation). Segmentation of regions of interest (ROI) can be performed manually, but such manual delineation is timeconsuming, highly variable and very tedious in 3-D. For these reasons, many computer aided PET segmentation techniques have been developped. These methods can be divided into four categories : thresholding, learning methods, stochastic models and variational approaches [1].

Among variational approaches, deformable models and most notably active contours, originally proposed by Kass

\footnotetext{
Work supported by European Union FP7-INMiND project http://www.uni-muenster.de/InMind/
}

and Witkin [2], are commonly used in medical imaging to delineate ROIs [3]. They are particularly appropriate for biological volume delineation because they guarantee contour integrity while most other methods do voxel-to-voxel selection, hence losing shape information. The extension of active contours to 3-D has been coined as active surfaces and their computer representation as active meshes. Active surfaces rely on the definition of external force fields computed from image information. Several expressions have been proposed to define noise robust force fields, most notably Gradient Vector Flow (GVF) [4], and more recently Vector Field Convolution (VFC) by Li and Acton [5], which have superior noise robustness than GVF fields. A VFC field is computed by convolving an edge map with a vector field kernel whose vectors are pointing towards the kernel centre with decreasing intensity with distance to centre. Despite these improvements, deformable models are often regarded as impractical in PET segmentation due to issues with initialisation and high sensitivity to noise [1]. Active surfaces in particular have been sparingly used in static PET [6] and, to our knowledge, no deformable model method dedicated to dynamic 3-D PET, exploiting the temporal redundancy of the signal along the frames, have been proposed.

We propose a new framework for dynamic PET image segmentation using edge-based parametric active surfaces. We define a vector-based gradient that is derived from an adaptive weighted structure tensor of the $3-D+t$ image to take full advantage of the temporal information along the acquisition. Our model performs 3-D segmentation of the kinetic profiles of voxels, hence reducing sensitivity to noise. We show that our 4-D framework significantly improves the result of the segmentation compared to single frame based 3-D models. We assess the performances of our method using numerical head phantoms and GATE Monte Carlo simulations.

\section{PROPOSED METHOD}

We propose a new 4-D active surface segmentation framework that extends classic vector field convolution to dynamic 3-D+t PET imaging. We call this method Multidimensional Vector Field Convolution (MVFC). 


\subsection{Notations}

We denote by $\mathbf{I}$ a dynamic PET sequence of $N$ temporal 3-D frames :

$$
\mathbf{I}(\vec{x}, t):\left(\Omega^{3} \times t\right) \in \mathbb{R}^{4} \rightarrow \mathbb{R},
$$

where $\Omega^{3}$ is the 3-D spatial domain of the PET image, $\vec{x}=$ $\left(x^{1}, x^{2}, x^{3}\right) \in \Omega^{3}$ are the voxel coordinates. We denote by $\mathbf{I}_{\mathbf{k}}$ the $k^{t h}$ temporal frame:

$$
\mathbf{I}(\vec{x}, t)=\left(\mathbf{I}_{\mathbf{1}}(\vec{x}), \ldots, \mathbf{I}_{\mathbf{k}}(\vec{x}), \ldots, \mathbf{I}_{\mathbf{N}}(\vec{x})\right) .
$$

We define our 3-D parametric active surface $\mathbf{S}^{t}$ at timestep $\mathrm{t}$ as a mapping of a bivariate parameter $(s, r)$ on a regular grid $\Omega^{s}$ superimposed on the spatial image domain $\Omega^{3}$.

$$
(s, r) \rightarrow \mathbf{S}^{t}(s, r)=\left[x^{1}(s, r), x^{2}(s, r), x^{3}(s, r)\right]^{T},
$$

where ${ }^{T}$ is the transposition operator.

\subsection{MVFC}

We define a new external force field for the active surface $\mathbf{S}^{t}$ at step $\mathrm{t}$ :

$$
\overrightarrow{\mathbf{F}}_{e x t}^{t}\left(\mathbf{S}^{t}\right)=\mathbf{N}_{+}^{\omega} * K,
$$

where $*$ is the convolution operation, and $\mathrm{K}$ is a vector field kernel (VFK), whose magnitude function controls the fading of the VFK intensities with distance to centre. In this work, we chose the magnitude function $m(x, y, z)=$ $(r+\epsilon)^{-\gamma}$, with $r$ the distance to centre, $\gamma>0$ the fading parameter, and $\epsilon$ a small positive number to avoid division by zero at VFK centre [5]. $\mathbf{N}_{+}^{\omega}$ is a vector-based gradient tailored for 4-D PET images defined hereafter.

Deformation of $\mathbf{S}^{t}$ is guided by the Euler-Lagrange equation, which can be seen as a force balance equation, and resolution is made through an iterative Euler scheme [2] :

$$
\frac{\partial \mathbf{S}^{t}}{\partial t}=\alpha \Delta \mathbf{S}^{t}-\beta \Delta^{2} \mathbf{S}^{t}+\overrightarrow{\mathbf{F}}_{e x t},
$$

where elasticity and rigidity terms are weighted by $\alpha$ and $\beta$.

\subsection{Gradient of a 4-D PET image}

In PET imaging, time activity curves (TAC) represent the kinetics of the tracer uptake in the body. We define a gradient that measures the difference of shape and magnitude of the TAC between tissues. It extends the work of Di Zenzo in 2D color images [7] to 3-D+t PET image sequences composed of $N$ temporal frames. We consider $\mathbf{I}$ as a $3 \mathrm{D} \rightarrow N$-D vector field, and we define a local vector geometry. The total differential $d \mathbf{I}$ of $\mathbf{I}$ is :

$$
d \mathbf{I}=\frac{\partial \mathbf{I}}{\partial x^{1}} d x^{1}+\frac{\partial \mathbf{I}}{\partial x^{2}} d x^{2}+\frac{\partial \mathbf{I}}{\partial x^{3}} d x^{3} .
$$

We define a new first fundamental form based on a weighted structure tensor that accounts for the underlying physics and physiology of 4-D PET imaging, as tissues are more or less contrasted with surrounding regions along time. Let $\mathbf{X}=\left(x^{1}, x^{2}, x^{3}\right)^{T}$, the new weighted quadratic form is defined as :

$$
\|d \mathbf{I}\|_{\omega}^{2}=d \mathbf{X}^{T} \mathbf{G}_{\omega} d \mathbf{X}
$$

where

$$
G_{\omega}=\sum_{k=1}^{N} \omega_{k}\left(\begin{array}{ccc}
\frac{\partial \mathbf{I}_{\mathbf{k}}^{2}}{\partial x^{1}} & \frac{\partial \mathbf{I}_{\mathbf{k}}}{\partial x^{1}} \frac{\partial \mathbf{I}_{\mathbf{k}}}{\partial x^{2}} & \frac{\partial \mathbf{I}_{\mathbf{k}}}{\partial x^{1}} \frac{\partial \mathbf{I}_{\mathbf{k}}}{\partial x^{3}} \\
\frac{\partial \mathbf{I}_{\mathbf{k}}}{\partial x^{2}} \frac{\partial \mathbf{I}_{\mathbf{k}}}{\partial x^{1}} & \frac{\partial \mathbf{I}_{\mathbf{k}}}{\partial x^{2}} & \frac{\partial \mathbf{I}_{\mathbf{k}}}{\partial x^{2}} \frac{\partial \mathbf{I}_{\mathbf{k}}}{\partial x^{3}} \\
\frac{\partial \mathbf{I}_{\mathbf{k}}}{\partial x^{3}} \frac{\partial \mathbf{I}_{\mathbf{k}}}{\partial x^{1}} & \frac{\partial \mathbf{I}_{\mathbf{k}}}{\partial x^{3}} \frac{\partial \mathbf{I}_{\mathbf{k}}}{\partial x^{2}} & \frac{\partial \mathbf{I}_{\mathbf{k}}}{\partial x^{3}}
\end{array}\right),
$$

where $\omega_{k}$ is a weighting factor of frame $\mathbf{I}_{k}$ defined hereafter, which accounts for the tracer dynamics in the body to improve the robustness of the method. Eigenvalues $\lambda_{i}=$ $\left(\lambda_{+}, \lambda_{-}^{1}, \lambda_{-}^{2}\right)$ associated with $G_{\omega}$ give the squared vectorbased gradient amplitude in the three directions of space in the local base of extremal variations, i.e. the extremal values of the weighted quadratic form of eq. (5). One eigenvalue, $\lambda_{+}$gives the maximum variation intensity, and the other two give variations in orthogonal directions. We use a gradient norm based on the three eigenvalues as proposed in [8] :

$$
\mathbf{N}_{+}^{\omega}=\sqrt{\lambda_{+}+\lambda_{-}^{1}+\lambda_{-}^{2}}=\sqrt{\operatorname{trace}\left(G_{\omega}\right)} .
$$

\subsection{Weighting factors computation}

As the contrast between tissues is varying along time, we propose to weight the influence of frames in the segmentation process, favouring the ones where the objects of interest can be better detected. At each iteration, we define $R_{k}^{i n t}$ as the set of voxels located inside $\mathbf{S}^{t}$ and $R_{k}^{\text {out }}$ as the set of voxels located between $\mathbf{S}^{t}$ and $\lambda \mathbf{S}^{t}$, where $\lambda \mathbf{S}^{t}$ is an isotropic dilation of $\mathbf{S}^{t}$. We limit $R_{k}^{\text {out }}$ to this dilation to reduce possible influence from further regions. $R_{k}^{\text {int }}$ can be seen as an estimation of the ROI volume at iteration $t$.

Average intensities in $R_{k}^{\text {int }}$ and $R_{k}^{\text {out }}$ differ in wellcontrasted frames. This information is used to define an estimator of contrast quality in frame $\mathbf{I}_{k}$. The weighting factor $\omega_{k}$ is then :

$$
\omega_{k}=\frac{\left|\overline{\mathbf{I}}_{k}^{\text {in }}-\overline{\mathbf{I}}_{k}^{\text {out }}\right|}{\sum_{j}\left|\overline{\mathbf{I}}_{j}^{\text {in }}-\overline{\mathbf{I}}_{j}^{\text {out }}\right|},
$$

where $\overline{\mathbf{I}}_{k}^{i n}$ is the average intensity ${ }^{1}$ in $R_{k}^{\text {int }}$, and $\overline{\mathbf{I}}_{k}^{\text {out }}$ the average intensity in $R_{k}^{\text {out }}$. Weights are recomputed at each timestep of the active surface evolution.

In Fig. $1, \mathbf{S}^{t}$ is a 2D representation of the active surface at timestep $t$ of the deformation process. On the left, in the first frame of the simulated PET sequence, the difference between inner and outer intensities is low. On the right, in the tenth frame, it is significant. The weights favour the gradient information of such well contrasted frames in the calculus of the structure tensor $G_{\omega}(6)$.

\footnotetext{
${ }^{1}$ The intensity in each frame is normalized between 0 and 1
} 

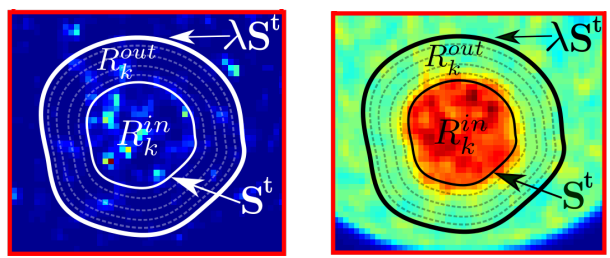

Fig. 1. 2D illustration of the weighting method. Left : low contrast frame. Right : high contrast frame.

\subsection{Numerical implementation}

We implemented our method using MATLAB. The numerical solution to eq. (3) is achieved using a discretised finite difference approach. We use an explicit triangulated mesh representation. The empirically selected VFK parameters for MVFC are: kernel size of 5 and fading power of 1.7.

\subsection{Initial shape prior to deformation}

We define the initial shape with the Poisson inverse gradient (PIG) algorithm [9]. This algorithm relies on an initial external force field, and Dirichlet boundary conditions. For initial surface calculation, we set $\omega_{k}=1, \forall k$ and compute the initial external force field as in eq. (2). We use high kernel size and low kernel attenuation so that the initial shape obtained after the PIG energy calculation is a coarse but noise-robust estimation of the region of interest.

\section{EXPERIMENTS}

\subsection{Simulations}

We performed GATE Monte Carlo simulations [10] of Philips Gemini GXL PET 4-D acquisitions and we focused on cerebellum segmentation of the Zubal head phantom [11]. TACs were generated according to a three-compartment model. The reconstruction of the dynamic PET image was performed using a fully 3-D OSEM iterative method into 20 frames of $2.2 \times 2.2 \times 2.8 \mathrm{~mm}^{3}$ voxels, using 10 iterations and 16 subsets (see Fig. 2(a)). All frames were smoothed with a Gaussian blur of $5 \mathrm{~mm}$.

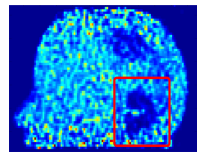

(a)

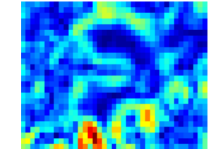

(b)

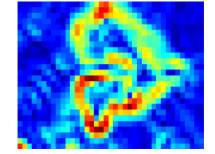

(c)

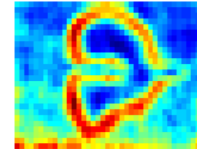

(d)
Fig. 2. (a) Sagittal slice of Monte Carlo GATE simulation in a representative frame. Red square : zoomed cerebellum area used in b,c,d. (b-c) gradient magnitude of two frames of the sequence, (d) proposed 4-D gradient magnitude $\mathbf{N}_{+}^{\omega}$.
Fig. 3. Representative similarity criteria, obtained on a dynamic PET image simulation. VFC for each frame (red) and MVFC 4-D result (green)

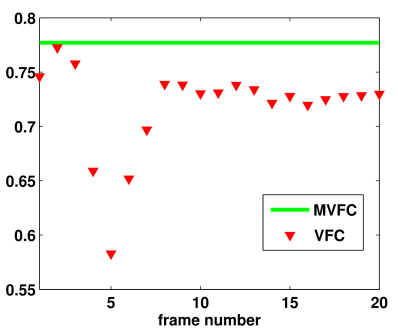

(a) Jaccard similarity index

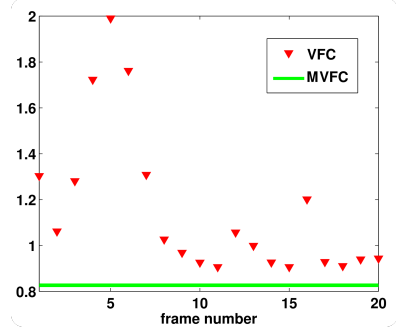

(b) average Hausdorff distance

\subsection{Comparison to other methods}

The proposed dynamic MVFC active surface method was compared with a classic VFC active surface method, calculated for each frame of the dynamic PET image. VFC was initialized with the initial shape calculated in MVFC using proposed vector-based gradient to avoid biasing the results. All else being equal, this static VFC method focused on one frame $\mathbf{I}_{k}$ of the dynamic PET image and used the classic gradient of intensity as the edge map, instead of the 4-D gradient $\mathbf{N}_{+}^{\omega}$ proposed in MVFC. We therefore obtained 20 results for classic VFC for each frame, that we compared to the single result obtained with MVFC. Figure 2 illustrates the differences between intensity gradients and the proposed vector-based gradient for dynamic PET image segmentation. The ground truth volumes were compared to our results by using two quantitative criteria : the Jaccard index as a measure of volume similarity [12] and the average Hausdorff distance [13] .

\section{RESULTS}

For both criteria, the MVFC method outperformed VFC results on all frames of the simulated images (Fig. 3). Mean Jaccard coefficient was 0.77 for MVFC while it was 0.71 for VFC. Average Hausdorff distance was 0.82 voxel for MVFC while it was 1.15 voxel for VFC. Figure 4(c) and 4(d) illustrate the MVFC result of the segmentation on one slice, where we observe good agreement with respect to ground truth. In all simulations, the estimated weights of the final active surface were in good agreement with the subjective quality of each frame. While the best results of single frame VFC were relatively close to the ones obtained with MVFC, the two criteria were not maximized in the same frames. On the opposite, a single active surface result was obtained for the 4-D PET sequence with MVFC, with better figure of merit scores. This can be explained by the different edge intensity measurements of the methods. This is illustrated in figure 2 which 


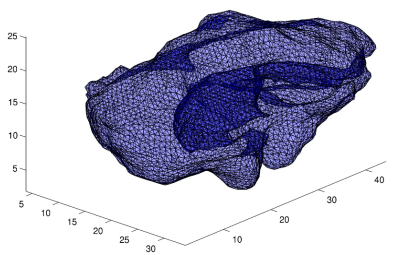

(c)

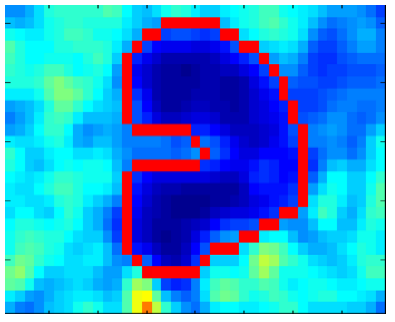

(e)

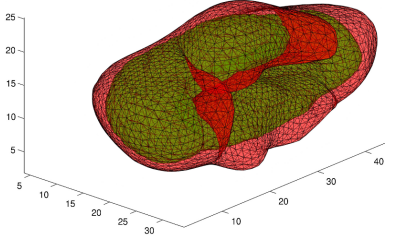

(d)

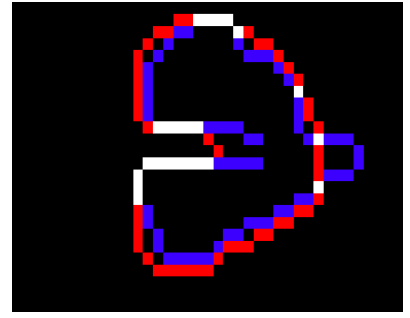

(f)
Fig. 4. Top : Computer mesh representation of cerebellum segmentation. (a) ground truth. (b) initial shape (green) and final shape (red) with MVFC.

Bottom : (c) contour of final mesh on one slice. (d) contour of final mesh (red) and contour of ground truth (blue), exact superposition (white).

shows edge maps calculated with classic scalar gradient (Fig. 2(b-c)) and with the proposed 4-D gradient $\mathbf{N}_{+}$(Fig. 2(d)). Moreover, both VFC and MVFC models shared the same initial shape, computed from the MVFC initial field to assess the intrinsic quality of the method rather than initialization quality. In fact, due to the poor quality of the gradient map in most of the frames of the PET sequence, the PIG initialization algorithm would not be appropriate for correct convergence of the segmentation, confirming the superiority of the proposed vector-based 4-D method for dynamic PET image segmentation.

\section{CONCLUSION}

We have proposed a novel vector-based method for the segmentation of dynamic PET images using active surfaces, based on a new structure tensor of the PET image and an efficient weighting scheme. Depending on the VFK size and its decreasing power parameter, VFC fields are more or less sensitive to distant and weak edges, making this method particularly suitable in noisy PET volume delineation. As we use both spatial and temporal features to estimate the kinetic transitions between voxels, our method is more robust to noise than static based methods. The proposed framework can be easily adapted to any vector-based image. Results on realistic simulations demonstrated the potential impact of the method for dynamic PET image segmentation.

\section{REFERENCES}

[1] H. Zaidi and I. El Naqa, "PET-guided delineation of radiation therapy treatment volumes: a survey of image segmentation techniques," European Journal of Nuclear Medicine and Molecular Imaging, vol. 37, no. 11, pp. 2165-2187, Mar. 2010.

[2] M. Kass, A. Witkin, and D. Terzopoulos, "Snakes: Active contour models," International journal of computer vision, vol. 1, no. 4, pp. 321-331, 1988.

[3] T. McInerney and D. Terzopoulos, "Deformable models in medical image analysis," in Mathematical Methods in Biomedical Image Analysis, 1996, pp. 171-180.

[4] C. Xu and J.L. Prince, "Snakes, shapes, and gradient vector flow," Image Processing, IEEE Transactions on, vol. 7, no. 3, pp. 359-369, mar 1998.

[5] B. Li and S. T. Acton, "Active contour external force using vector field convolution for image segmentation," IEEE Transactions on Image Processing, vol. 16, no. 8, pp. 2096-2106, Aug. 2007.

[6] J. Tohka, "Surface extraction from volumetric images using deformable meshes: a comparative study," Computer Vision ECCV 2002, pp. 127-145, 2002.

[7] S. Di Zenzo, "A note on the gradient of a multi-image," Computer Vision, Graphics, and Image Processing, vol. 33, no. 1, pp. 116-125, 1986.

[8] D. Tschumperle and R. Deriche, "Diffusion pdes on vector-valued images," Signal Processing Magazine, IEEE, vol. 19, no. 5, pp. 16-25, sep 2002.

[9] B. Li and S.T. Acton, "Automatic active model initialization via poisson inverse gradient," IEEE Transactions on Image Processing, vol. 17, no. 8, pp. 1406-1420, Aug. 2008.

[10] S Jan et al., "Gate: a simulation toolkit for pet and spect," Physics in Medicine and Biology, vol. 49, no. 19, pp. 4543, 2004.

[11] G. Zubal et al, "Computerized three-dimensional segmented human anatomy," Medical Physics, vol. 21, no. 2, pp. 299-302, 1994.

[12] P. Jaccard, La distribution de la flore dans la zone alpine, Armand Colin, 1907.

[13] M.-P. Dubuisson and A.K. Jain, "A modified hausdorff distance for object matching," in Pattern Recognition, 1994. Image Processing., Proceedings of the 12th IAPR International Conference on, oct 1994, vol. 1, pp. 566568. 